NISTIR 8027

\title{
Stable Explicit Time Marching in Well-Posed or Ill-Posed Nonlinear Parabolic Equations
}

\author{
Alfred S. Carasso \\ Applied and Computational Mathematics Division \\ Information Technology Laboratory
}

This publication is available free of charge from:

http://dx.doi.org/10.6028/NIST.IR.8027

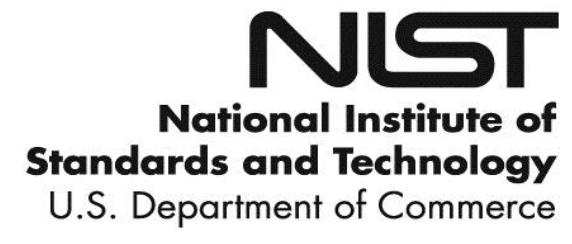




\title{
Stable Explicit Time Marching in Well-Posed or Ill-Posed Nonlinear Parabolic Equations
}

\author{
Alfred S. Carasso \\ Applied and Computational Mathematics Division \\ Information Technology Laboratory
}

This publication is available free of charge from: http://dx.doi.org/10.6028/NIST.IR.8027

October 2014

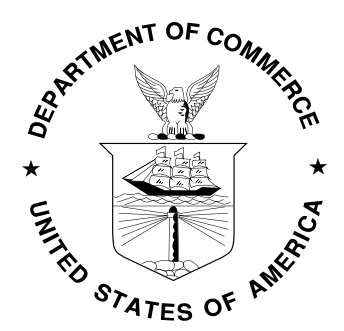

U.S. Department of Commerce Penny Pritzker, Secretary

National Institute of Standards and Technology Willie May, Acting Under Secretary of Commerce for Standards and Technology and Acting Director 


\title{
STABLE EXPLICIT TIME MARCHING IN WELL-POSED OR ILL-POSED NONLINEAR PARABOLIC EQUATIONS
}

\author{
ALFRED S. CARASSO*
}

\begin{abstract}
This paper analyzes an effective technique for stabilizing pure explicit time differencing in the numerical computation of multidimensional nonlinear parabolic equations. The method uses easily synthesized linear smoothing operators at each time step to quench the instability. Smoothing operators based on positive real powers of the negative Laplacian are helpful, and $(-\Delta)^{p}$ can be realized efficiently in rectangular domains using FFT algorithms. The stabilized explicit scheme requires no Courant restriction on the time step $\Delta t$, and is of great value in computing well-posed parabolic equations on fine meshes, by simply lagging the nonlinearity at the previous time step. Such stabilization leads to a distortion away from the true solution. However, that error is often small enough to allow useful results in many problems of interest.

The stabilized explicit scheme is also stable when run backward in time. This allows for relatively easy and useful computation of a significant class of multidimensional nonlinear backward parabolic equations, and complements the quasi-reversibility method. In the canonical case of linear autonomous selfadjoint backward parabolic equations, with solutions satisfying prescribed bounds, it is proved that the stabilized explicit scheme can produce results that are nearly best-possible. Such backward reconstructions are of increasing interest in environmental forensics, where contaminant transport is often modeled by advection dispersion equations.

The paper uses fictitious mathematically blurred $512 \times 512$ pixel images as illustrative examples. Such images are associated with highly irregular jagged intensity data surfaces that can severely challenge ill-posed nonlinear reconstruction procedures. Instructive computational experiments demonstrate the capabilities of the method in $2 \mathrm{D}$ rectangular regions.
\end{abstract}

Key words. FFT Laplacian stabilization, forward or backward nonlinear parabolic equations; non-integer power Laplacian; nonlinear image deblurring; quasi-reversibility method; stabilized explicit scheme; Van Cittert iteration.

AMS subject classifications. 35K55, 35R25, 65M06, 65M30, 68U10.

1. Introduction. This paper develops an effective technique for stabilizing pure explicit time differencing in the numerical computation of multidimensional nonlinear parabolic equations. As is well-known [21, p. 208], while explicit schemes are highly desirable because of their simplicity, such schemes are seldom used as they entail prohibitive Courant stability restrictions on the time step $\Delta t$. Rather, unconditionally stable implicit schemes are commonly used, even though such schemes require sophisticated and computationally intensive solutions of the resulting algebraic systems of difference equations at each time step. The present stabilized explicit scheme requires no Courant restriction on $\Delta t$, and hence would be of great value in computing multidimensional, well-posed, nonlinear parabolic equations on fine meshes, by simply lagging the nonlinearity at the previous time step.

For ill-posed backward parabolic equations, classical stepwise time marching algorithms, whether explicit or implicit, are necessarily unconditionally unstable, [21, p. 59]. However, remarkably, the stabilized explicit scheme is stable even when run backward in time. As will be shown in the examples below, this scheme opens the door to relatively easy and useful computation of multidimensional nonlinear backward parabolic equations, a category of problems that is widely believed to be intractable. The method complements the quasi-reversibility method [13], whose formulation is primarily oriented toward linear problems. There is considerable interest in such backward reconstructions, notably in the developing field of environmental forensics

* Applied and Computational Mathematics Division, National Institute of Standards and Technology, Gaithersburg, MD 20899. (alfred.carasso@nist.gov). 
[3], [4], [17], [23], where contaminant transport is often modeled by parabolic advection dispersion equations [14], [22]. However, the class of nonlinear problems that can be handled by the present method is limited, although it is significant.

Stabilization of the explicit scheme is accomplished by applying an easily synthesized linear smoothing operator at each time step to quench the instability. Smoothing operators based on positive non-integer powers of the negative Laplacian have been found helpful. Inevitably, such intervention leads to a distortion away from the true solution. This is the stabilization penalty. The scheme is useful on a given problem only to the extent that the accompanying distortion is sufficiently small. When this is the case, the method is an effective preliminary exploration tool that can produce valuable information in quite difficult problems. Such information can subsequently be confirmed using more elaborate computational methods. As noted below, the method assumes the validity of Eq. (2.4). That hypothesis appears to be verified in numerous computational experiments.

While the computation of nonlinear problems is the principal aim of this work, as well as the focus of the illustrative examples, valuable insight is provided by the analysis in Sections 2 through 5, which addresses a companion class of linear problems. Consideration of the transparent linear case enables isolating the stabilization penalty, evaluating its significance, and interpreting it as a form of regularization. In the canonical case of linear autonomous selfadjoint backward parabolic equations with solutions satisfying prescribed bounds [10], [15], a sharp estimate for the uncertainty in reconstruction can be obtained using logarithmic convexity arguments. The analysis in Eqs. (3.18-3.19) shows that the error in the stabilized explicit scheme differs from that fundamental uncertainty only by the stabilization penalty. Moreover, it is shown that that penalty can be expected to be small in a wide class of parabolic problems.

The present paper complements and extends earlier work discussed in [7], by providing the analytical justification not included in [7], by considering the wellposed forward problem as well as the backward problem, and by exhibiting successful computation of nonlinear problems for which the competing Van Cittert method, previously developed and applied in [7], fails.

1.1. Use of fictitious images as illustrative test examples. Images can provide useful and challenging computational test examples for 2D nonlinear parabolic equations. Using a sharp image as initial data in a well-posed forward parabolic equation results in a fictitious blurred image, yet one in which the effects of various nonlinearities can be easily gauged with the naked eye. Using a mathematically blurred image as input data in the ill-posed backward parabolic problem, poses a severe test for an inverse reconstruction algorithm. As shown in Figure 1.1, highly complicated intensity data are typically associated with images of familiar objects. Such complex yet meaningful data are not easily generated analytically by combining mathematical formulae. Also, given a familiar object, the degree of success in nonlinear backward reconstruction is often immediately apparent. On the other hand, comparing the reconstructed intensity surface with the original intensity surface may not always be helpful; a slight blurring of the image, one that does not affect object recognition, can cause nonintuitive changes in the original surface.

Very little is known either analytically or computationally about nonlinear backward parabolic equations. Examples of physically plausible yet false reconstructions have been found [6]. Given a specific nonlinear equation, preliminary exploration of the associated fictitious image deblurring problem provides valuable information about that equation, even when the intended scientific or engineering application is 


\section{Images are associated with complicated surface plots of intensity values}

\section{Shirley Temple}

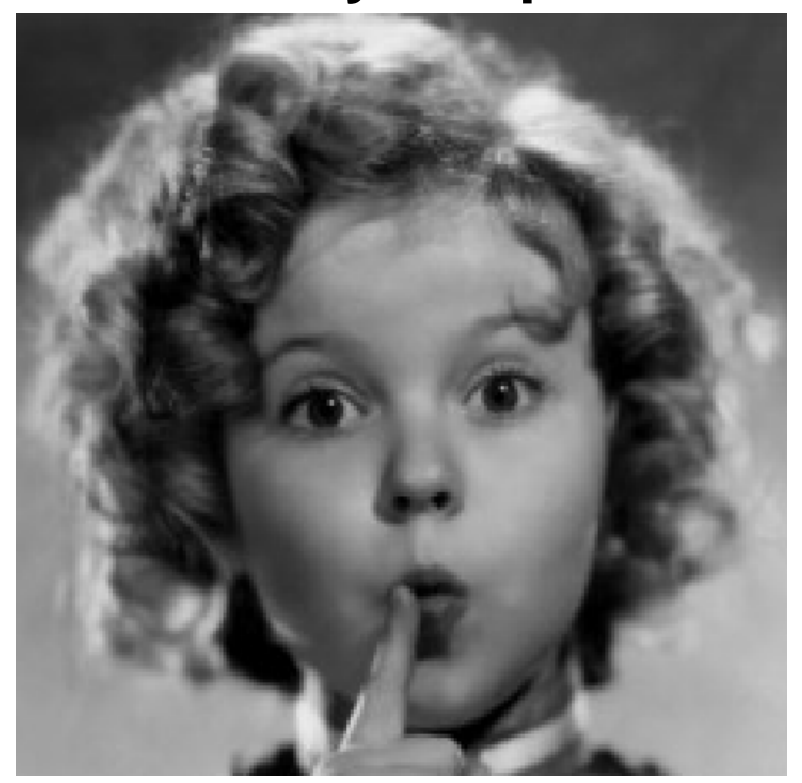

\section{Pyramids at Giza}

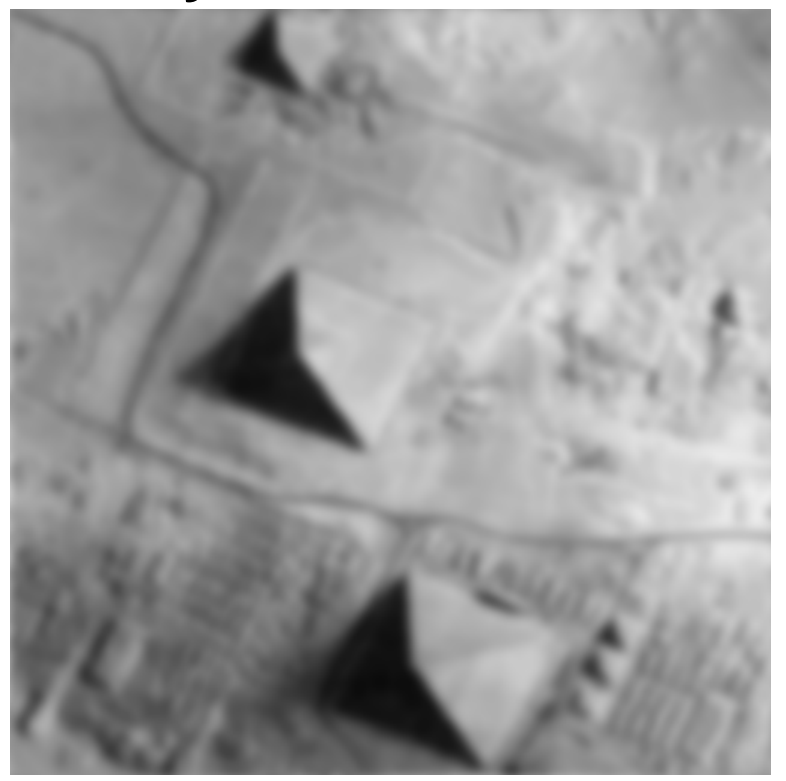

\section{Plot of intensity values}

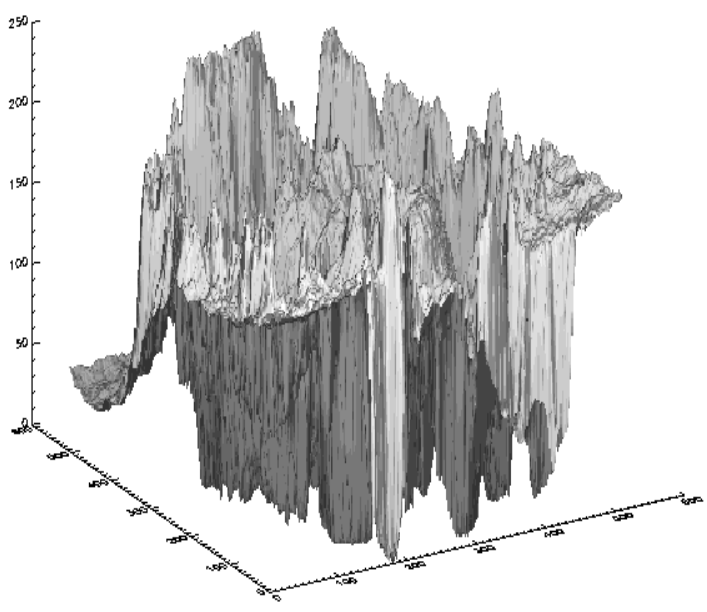

\section{Plot of intensity values}

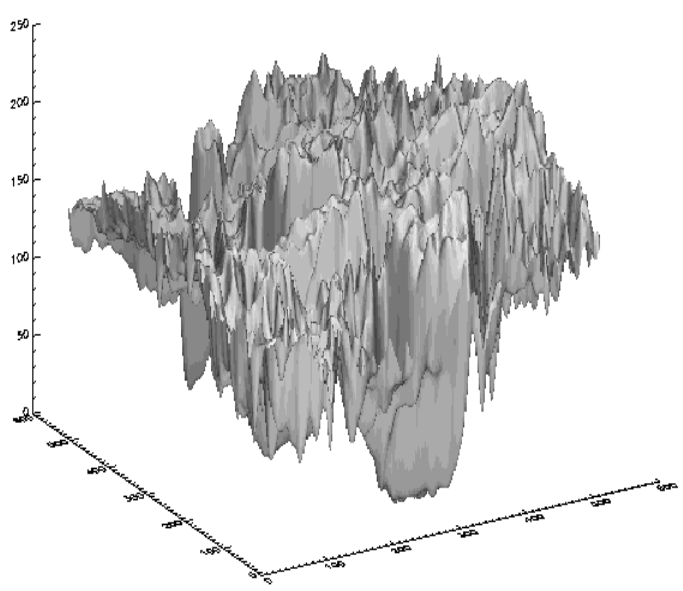

FIG. 1.1. Reconstruction of images requires recovery of highly irregular, jagged intensity data surfaces that severely challenge ill-posed computational algorithms. Accordingly, fictitious nonlinearly blurred images provide instructive test examples in this paper. 
unrelated to imaging. Accordingly, images will be used as test examples to illustrate the merits of the stabilized explicit scheme.

2. A companion class of autonomous linear second order parabolic equations. Useful insight into the nonlinear computations can be gained by considering the following class of problems. Let $L$ be a linear autonomous second order elliptic differential operator with smooth variable coefficients in a region $\Omega$ in $R^{n}$, with homogeneous Dirichlet or Neumann boundary conditions on $\partial \Omega$. Let \|\|$_{2}$ denote the norm on $L^{2}(\Omega)$. We assume that $L$ has a complete set of orthonormal eigenfunctions $\left\{\phi_{m}\right\}_{m=1}^{\infty}$ in $L^{2}(\Omega)$, corresponding to eigenvalues $\left\{\lambda_{m}\right\}_{m=1}^{\infty}$ satisfying

$$
0 \leq\left|\lambda_{1}\right| \leq\left|\lambda_{2}\right| \leq \cdots \leq\left|\lambda_{m}\right| \cdots \uparrow \infty
$$

The eigenvalues $\lambda_{m}$ of $L$ need not all be of the same sign, and the evolution equation

$$
u_{t}=-L u, \quad t>0, \quad u(0)=f,
$$

may not be well-posed. However, we assume that if a solution exists in Eq. (2.2), it is unique.

We shall also consider the auxiliary well-posed parabolic equation on $\Omega$

$$
v_{t}=\Delta v, \quad t>0, \quad v(0)=f,
$$

with the same homogeneous boundary conditions on $\partial \Omega$ as in Eq. (2.2). The Laplacian operator is selfadjoint and isotropic, whereas this may not be the case with the differential operator $L$. Let $L^{*}$ denote the adjoint of $L$. With real $p, q>1$, we make the following assumption regarding the semigroups generated by $-(-\Delta)^{p}$ and $-\left(L^{*} L\right)^{q / 2}$ : Given any $\omega>0$, and $q$ with $1<q \leq 3$, there exist constants $\Gamma, \epsilon>0$, and $p \geq q$, such that for all $g \in L^{2}(\Omega)$ and sufficently small time step $\Delta t$,

$$
\left\|\exp \left\{-\epsilon \Delta t(-\Delta)^{p}\right\} g\right\|_{2} \leq \Gamma\left\|\exp \left\{-\omega \Delta t\left(L^{*} L\right)^{q / 2}\right\} g\right\|_{2} .
$$

The linear operator $H=\left(\exp \left\{-\epsilon \Delta t(-\Delta)^{p}\right\}\right)\left(\exp \left\{\omega \Delta t\left(L^{*} L\right)^{q / 2}\right\}\right)$ is well-defined on the range of $\left(\exp \left\{-\omega \Delta t\left(L^{*} L\right)^{q / 2}\right\}\right)$, which is dense in $L^{2}(\Omega)$. The inequality in Eq. (2.4) would follow if it can be shown that $H$ can be extended to a bounded operator on all of $L^{2}(\Omega)$, with $\|H\|_{2} \leq \Gamma$.

Eq. (2.4) appears to be validated in numerous computational experiments. Results of a similar nature are known in the theory of Gaussian estimates for heat kernels. See e.g. [1], [2], [18], [19], and the references therein.

3. Stabilizing the pure explicit scheme. The discussion here will focus exclusively on time discretizations, and the space variables will remain continuous in all the equations below. For the equation $u_{t}=-L u, \quad 0 \leq t \leq T$, in Eq. (2.1), the pure explicit scheme, $u^{n+1}=u^{n}-\Delta t L u^{n}, n=0,1,2, \cdots, N_{T}-1, \Delta t=T / N_{T}$, is a first order accurate scheme, requiring a stringent stability restriction on the time step $\Delta t$, when Eq. (2.1) represents a forward, well-posed, multidimensional parabolic equation [21]. For this reason, the pure explicit scheme is seldom used. If $u(x, t)$ is the exact unique solution of $u_{t}=-L u$, then $u^{n+1}=u^{n}-\Delta t L u^{n}+\tau^{n}$, where $\tau^{n}=1 / 2(\Delta t)^{2}\left(L^{2} u\right)(\tilde{t})$, with the intermediate time value $\tilde{t}$ lying between $n \Delta t$ and $(n+1) \Delta t$. The quantity $\tau^{n}$ is the truncation error.

With fixed $\omega, \Delta t$, and $q, 1<q \leq 3$, let $S$ and $A$ denote the linear operators in $L^{2}(\Omega)$ defined by

$$
S=\exp \left\{-\omega \Delta t\left(L^{*} L\right)^{q / 2}\right\}, \quad A=S(I-\Delta t L) .
$$


We shall consider a compensated pure explicit scheme for $u_{t}=-L u, t>0, u(0)=f$, defined as follows

$$
w^{0}=f
$$$$
w^{n+1}=A w^{n} \equiv S\left\{w^{n}-\Delta t L w^{n}\right\}, \quad n=0,1,2, \cdots, N_{T}-1, \quad \Delta t=T / N_{T} .
$$

Lemma 1. Let $\left\{\lambda_{m}\right\}_{m=1}^{\infty}$ be the eigenvalues of $L$ satisfying Eq. (2.1). With real $q>1$, fix a positive integer $Q$ such that $\left|\lambda_{Q}\right|>0$, and choose $\omega \geq\left|\lambda_{Q}\right|^{1-q}$. Then,

$$
\left(1+\Delta t\left|\lambda_{m}\right|\right) \exp \left\{-\omega \Delta t\left|\lambda_{m}\right|^{q}\right\} \leq 1+\Delta t\left|\lambda_{Q}\right|, \quad m \geq 1 .
$$

Proof: The inequality in Eq. (3.3) is valid for $1 \leq m \leq Q$ in view of Eq. (2.1). For $m>Q$,

$$
\exp \left\{-\omega \Delta t\left|\lambda_{m}\right|^{q}\right\} \leq \exp \left\{-\omega \Delta t\left|\lambda_{m}\right|\left|\lambda_{Q}\right|^{q-1}\right\} \leq \exp \left\{-\Delta t\left|\lambda_{m}\right|\right\}
$$

since $\omega\left|\lambda_{Q}\right|^{q-1} \geq 1$. Also, $\exp \left\{-\Delta t\left|\lambda_{m}\right|\right\} \leq\left(1+\Delta t\left|\lambda_{m}\right|\right)^{-1}$, since $1+x \leq e^{x}$ for real $x$. Hence, for $m>Q,\left(1+\Delta t\left|\lambda_{m}\right|\right) \exp \left\{-\omega \Delta t\left|\lambda_{m}\right|^{q}\right\} \leq 1$. QED.

Lemma 2. Let $q, Q, \omega$, be as in Lemma 1. Then, $\|A\|_{2} \leq 1+\Delta t\left|\lambda_{Q}\right|$, the compensated pure explicit scheme Eq. (3.2) is unconditionally stable, and $w^{n}$ satisfies

$$
\left\|w^{n}\right\|_{2} \leq \exp \left\{\left|\lambda_{Q}\right| n \Delta t\right\}\|f\|_{2}, \quad n=0,1,2, \cdots, N_{T} .
$$

Proof : Expanding in the orthonormal eigenfunctions $\left\{\phi_{m}(x, y)\right\}_{m=1}^{\infty}$ of $L$, let $w_{m}^{n}=<w^{n}, \phi_{m}>$. Then,

$$
w^{n+1}=\sum_{m=1}^{\infty} \exp \left\{-\omega \Delta t\left|\lambda_{m}\right|^{q}\right\}\left(1-\Delta t \lambda_{m}\right) w_{m}^{n} \phi_{m} .
$$

Hence, from Parseval's relation and Lemma 1,

$$
\begin{aligned}
\left\|w^{n+1}\right\|_{2} & \leq \sup _{m}\left\{\left(1+\Delta t\left|\lambda_{m}\right|\right) \exp \left[-\omega \Delta t\left|\lambda_{m}\right|^{q}\right]\right\}\left\|w^{n}\right\|_{2}, \\
& \leq\left(1+\Delta t\left|\lambda_{Q}\right|\right)^{n+1}\|f\|_{2} \leq \exp \left\{\left|\lambda_{Q}\right|(n+1) \Delta t\right\}\|f\|_{2} .
\end{aligned}
$$

QED.

Lemma 3. Let ||$|h| \|_{2, \infty}$ denote

$$
\||| h \mid\|_{2, \infty}=\sup _{0 \leq t \leq T}\left\{\|h(., t)\|_{2}\right\},
$$

and let $u(t)$ be the unique solution of $u_{t}=-L u, u(0)=f$, on $0 \leq t \leq T$. Then, with $S$ as in Eq.(3.1),

$$
\begin{aligned}
\left\|\tau^{n}\right\|_{2} & \leq 1 / 2(\Delta t)^{2}\left\|L^{2} u\right\| \|_{2, \infty}, \\
\left\|(I-S) u^{n}\right\|_{2} & \leq \omega \Delta t\left\|\left(L^{*} L\right)^{q / 2} u\right\|_{2, \infty}, \\
\Delta t\left\|(I-S) L u^{n}\right\|_{2} & \leq \omega(\Delta t)^{2}\|\|\left(L^{*} L\right)^{q / 2} L u \|_{2, \infty} .
\end{aligned}
$$


Proof: The inequality for the truncation error $\tau^{n}$ in Eq. (3.9) follows from Taylor's formula. Expanding in the orthonormal eigenfunctions $\left\{\phi_{m}(x, y)\right\}_{m=1}^{\infty}$ of $L$, and using $1-e^{x} \leq-x$ for real $x$, we have, with $u_{m}^{n}=<u^{n}, \phi_{m}>$

$$
\begin{aligned}
\left\|(I-S) u^{n}\right\|_{2}^{2} & =\sum_{m=0}^{\infty}\left(1-e^{-\omega \Delta t\left|\lambda_{m}\right|^{q}}\right)^{2}\left|u_{m}^{n}\right|^{2} \leq \sum_{m=0}^{\infty}\left(\omega \Delta t\left|\lambda_{m}\right|^{q}\right)^{2}\left|u_{m}^{n}\right|^{2} \\
& =(\omega \Delta t)^{2}\left\|\left(L^{*} L\right)^{q / 2} u^{n}\right\|_{2}^{2} \leq(\omega \Delta t)^{2}\left\|\left(L^{*} L\right)^{q / 2} u \mid\right\|_{2, \infty}^{2} .
\end{aligned}
$$

This proves the second inequality in Eq. (3.9), as well as the last inequality which is a corollary of the second. QED.

TheOrem 1. Let $u^{n}$ be the unique solution of $u_{t}=-L u, u(0)=f$, at $t=n \Delta t$. Let $w^{n}$ be the corresponding solution of the $S$-compensated scheme in Eq. (3.2), and let $E S(t) \equiv u^{n}-w^{n}$, denote the error at $t=n \Delta t, \quad n=0,1,2, \cdots, N_{T}$. Then, with $q, Q, \omega$, as in Lemma 1, and $\|||\|_{2, \infty}$ as in Eq. (3.8),

$\|E S(t)\|_{2} \leq e^{\left|\lambda_{Q}\right| t}\|E S(0)\|_{2}+\left\{\left(e^{\left|\lambda_{Q}\right| t}-1\right) /\left|\lambda_{Q}\right|\right\} \times$

$$
\left\{\omega\left\|\left|\left(L^{*} L\right)^{q / 2} u\left\|_{2, \infty}+\omega \Delta t\right\|\right|\left(L^{*} L\right)^{q / 2} L u\right\|_{2, \infty}+(\Delta t / 2)\left\|\mid L^{2} u\right\| \|_{2, \infty}\right\} .
$$

Proof: Let $g^{n}=\tau^{n}+(I-S) u^{n}+\Delta t(I-S) L u^{n}$. Then, $u^{n+1}=A u^{n}+g^{n}$, while $w^{n+1}=A w^{n}$. Therefore

$$
E S^{n+1}=u^{n+1}-w^{n+1}=A^{n} e^{n}+g^{n}=A^{n+1} E S^{0}+\Delta t \sum_{j=0}^{n} A^{n-j} g^{j} /(\Delta t)
$$

Hence, using Lemma 2 with $t=(n+1) \Delta t$,

$$
\begin{aligned}
\|E S(t)\|_{2} & \leq e^{\left|\lambda_{Q}\right| t}\|E S(0)\|_{2}+\left\{\left|\|g \mid\|_{2, \infty} / \Delta t\right\} \Delta t \sum_{j=0}^{n}\left\|A^{n-j}\right\|_{2},\right. \\
& \leq e^{\left|\lambda_{Q}\right| t}\|E S(0)\|_{2}+\left\{\left|\|g \mid\|_{2, \infty} / \Delta t\right\} \int_{0}^{t} e^{\left|\lambda_{Q}\right|(t-u)} d u\right. \\
& =e^{\left|\lambda_{Q}\right| t}\|E S(0)\|_{2}+\left\{\left|\|g \mid\|_{2, \infty} / \Delta t\right\}\left(e^{\left|\lambda_{Q}\right| t}-1\right) /\left|\lambda_{Q}\right| .\right.
\end{aligned}
$$

Next, using Lemma 3 to estimate $\left\{\left.|||g|\right|_{2, \infty} / \Delta t\right\}$, we obtain Eq. (3.11) from Eq. (3.13). QED.

3.1. The stabilization penalty in the well-posed forward problem. Consider the case of the well-posed forward parabolic problem $u_{t}=-L u, t>0, u(0)=f$, with $L$ a non-negative autonomous selfadjoint elliptic operator, and the given initial data $f(x)$ known exactly, or with high accuracy. Then, $E S(0)=0$ in Eq. (3.11). Choosing $\omega=\left|\lambda_{Q}\right|^{1-q}$, there remains a non-vanishing residual in Eq. (3.11), the stabilization penalty, as $\Delta t \downarrow 0$,

$$
\|E S(t)\|_{2} \leq\left(e^{\left|\lambda_{Q}\right| T}-1\right)\left\|\left.\left(L^{*} L\right)^{q / 2} u\left|\|_{2, \infty} /\right| \lambda_{Q}\right|^{q}+O(\Delta t), \quad 0 \leq t \leq T .\right.
$$

Clearly, if $T$ is large, the accumulated distortion may become unacceptably large as $t \uparrow T$, and the stabilized explicit scheme is not useful in that case. On the other 
hand, if $T$ is small, as is the case in many multidimensional problems of interest involving small values of $t$, it may be possible to choose $q>2$ and large $\left|\lambda_{Q}\right|$, with small enough $\left|\lambda_{Q}\right| T$ such that $\left(e^{\left|\lambda_{Q}\right| T}-1\right) /\left|\lambda_{Q}\right|^{q}$ is quite small. In that case, the stabilization penalty remains acceptably small on $0 \leq t \leq T$. We give several such examples below.

3.2. The stabilization penalty in the ill-posed backward problem. In the ill-posed backward parabolic case where $L$ is a non-positive self-adjoint operator, the class of initial values for which there exists a solution is very restricted. Here, the given initial data $f$ is seldom a highly accurate approximation to that unknown restricted data, and the input data error contribution in Eq. (3.11) cannot be neglected. With $\omega=\left|\lambda_{Q}\right|^{1-q}$, this leads to

$$
\|E S(t)\|_{2} \leq e^{\left|\lambda_{Q}\right| t}\|E S(0)\|_{2}
$$

Again, with large $T$, the non-vanishing residual in Eq. (3.15) will lead to large errors as $t \uparrow T$, and the backward scheme is not useful in such cases. For problems with small $T$, Eq. (3.15) is more interesting. Let the given initial data $f(x)$ approximate the unknown true data $u(x, 0)$ to within $\delta>0$ in the $L^{2}$ norm, and let the exact solution $u(., t)$ of the backward problem satisfy a known prescribed $L^{2}$ bound $M$ at $t=T$. Thus,

$$
\|E S(0)\|_{2} \leq \delta, \quad\|u(., T)\|_{2} \leq M .
$$

A judicious choice for $\left|\lambda_{Q}\right|$ is then

$$
\left|\lambda_{Q}\right|=(1 / T) \log (M / \delta)
$$

This leads to

$$
\begin{aligned}
\|E S(t)\|_{2} & \leq M^{t / T} \delta^{(T-t) / T} \\
& +\left\{\left(e^{\left|\lambda_{Q}\right| t}-1\right) /\left|\lambda_{Q}\right|^{q}\right\}\left\|\left|\left\|\left(L^{*} L\right)^{q / 2} u\right\|\right|_{2, \infty}+O(\Delta t), \quad 0 \leq t \leq T .\right.
\end{aligned}
$$

The first term on the right in Eq. (3.18) is best-possible, and represents the fundamental uncertainty in solutions of autonomous selfadjoint backward parabolic equations, satisfying a prescribed $L^{2}$ bound $M$, given initial data with $L^{2}$ error at most $\delta$, [10], [15]. The stabilization penalty augments this basic logarithmic convexity error bound by the addition of the second term in Eq. (3.18). In many problems of interest this added term can be quite small. For example, with parameter values such as $T=10^{-3}, M=10^{2}, \delta=10^{-4}, q=2.5$, we have $M / \delta=10^{6}, \log (M / \delta)=13.816$. From Eq. (3.17), $e^{\left|\lambda_{Q}\right| T}=10^{6},\left|\lambda_{Q}\right|=13816$. Hence, $\left|\lambda_{Q}\right|^{q}=2.244 \times 10^{10}, \omega=$ $\left|\lambda_{Q}\right|^{1-q}=6.16 \times 10^{-7}$. We then obtain from Eq. (3.18),

$$
\begin{aligned}
\|E S(t)\|_{2} & <M^{t / T} \delta^{(T-t) / T} \\
& +\left(4.46 \times 10^{-5}\right)\|\|\left(L^{*} L\right)^{q / 2} u \|_{2, \infty}+O(\Delta t), \quad 0 \leq t \leq T .
\end{aligned}
$$

Remark 1. The above analysis is valid in general domains $\Omega \in R^{n}$, and is helpful in the computation of nonlinear problems in the following way. If $L$ is a useful linear representative for the nonlinear spatial differential operator $\widetilde{L}$, one may synthesize the 
compensating operator $S$ in Eq. (3.1) by precomputing a sufficiently large number $K$ of eigenpairs $\left\{\lambda_{m}, \phi_{m}\right\}$ of the linear elliptic operator $L$, spanning a subspace $V_{K}$ in $L^{2}(\Omega)$. See e.g. [5], [8], [20]. Such a time-consuming task may pay large dividends if the resulting stabilized explicit scheme can actually be successfully applied to multidimensional nonlinear parabolic equations, equations with solutions well-approximated by elements of $V_{K}$. Expanding in the $K$ precomputed eigenfunctions of $L$, we may approximate the compensating operator $S$ in Eq. (3.1) as follows for $v \in V_{K} \subset L^{2}(\Omega)$,

$$
S v=\sum_{m=1}^{K} \exp \left\{-\omega \Delta t\left|\lambda_{m}\right|^{q}\right\}\left(\int_{\Omega} v(x) \phi_{m}(x) d x\right) \phi_{m}(x) .
$$

In a practical application, the parameters $\omega>0$ and $q>1$ in Eq. (3.20) must first be selected and adjusted interactively, based on prior knowledge about the solution. Such adjustment is similar to the manual tuning of an FM station, or the focusing of binoculars. A priori knowledge about the solution is a fundamental requirement in illposed reconstruction, and such knowledge is often found valuable even in well-posed computations.

Having located a successful pair $(\omega, q)$, define $\left|\lambda_{Q}\right|=\omega^{1 /(1-q)}$. In the well-posed forward problem, one may then use Eq. (3.14) to estimate the stabilization penalty as $\Delta t \downarrow 0$, in the successfully completed computation. However, that penalty is the one that would have been incurred with the linear representative operator $L$, rather than the actual nonlinear operator $\widetilde{L}$.

In the ill-posed backward problem, interactive adjustment of the parameter pair $(\omega, q)$ based on prior knowledge, is even more crucial in obtaining useful reconstructions as the values of $M$ and $\delta$ in Eq. (3.16) are seldom known accurately. Again, after locating an optimal pair, define $\left|\lambda_{Q}\right|=\omega^{1 /(1-q)}$. One may then use the second term in Eq. (3.18) to evaluate the stability penalty deviation from the best-possible result as $\Delta t \downarrow 0$. As before, this is the deviation that would have been incurred with $L$ rather than the actual $\widetilde{L}$.

4. Laplacian stabilization of the explicit scheme for $u_{t}=-L u$. Closed form expressions for the eigenfunctions of the Laplacian are known for specific domains that are important in applications, including rectangles, circles, and spheres [11], [16]. On such domains, the use of smoothing operators based on the Laplacian, rather than $L^{*} L$, to stabilize the explicit scheme for $u_{t}=-L u$, is of considerable interest. Such stabilization is feasible for parabolic problems $u_{t}=-L u$ for which the inequality in Eq. (2.4) holds.

With fixed constants $\Gamma, \epsilon, \Delta t$, and $p, 1<p \leq 3$, let $R$ and $B$ denote the linear operators in $L^{2}(\Omega)$ defined by

$$
R=\exp \left\{-\epsilon \Delta t(-\Delta)^{p}\right\}, \quad B=R(I-\Delta t L) / \Gamma .
$$

As in Eq. (3.20), let $\left\{\sigma_{m}, \psi_{m}\right\}_{m=1}^{K}$ denote a sufficiently large set of known eigenpairs of $(-\Delta)$ on $\Omega$, with the same boundary conditions on $\partial \Omega$ as prescribed for $L$. Let the subspace $V_{K} \subset L^{2}(\Omega)$ spanned by $\left\{\psi_{m}\right\}_{m=1}^{K}$ be large enough to well-approximate the solutions of $u_{t}=-L u$. We may then approximate the compensating operator $R$ on $V_{K}$ by

$$
R v=\sum_{m=1}^{K} \exp \left\{-\epsilon \Delta t\left|\sigma_{m}\right|^{p}\right\}\left(\int_{\Omega} v(x) \psi_{m}(x) d x\right) \psi_{m}(x), \quad v \in V_{K} .
$$


Using $R$ and $B$ leads to a new compensated explicit scheme for $u_{t}=-L u, u(0)=$ $f$, on $0 \leq t \leq T$,

$$
\begin{aligned}
& v^{0}=f \\
& v^{n+1}=B v^{n} \equiv R\left\{v^{n}-\Delta t L v^{n}\right\} / \Gamma, \quad n=0,1,2, \cdots, N_{T}-1, \quad \Delta t=T / N_{T} .
\end{aligned}
$$

Lemma 4. Let $q, Q, \omega$, be as in Lemma 1 , and let $R$ and $B$ be the operators in Eq. (4.1). Choose $\Gamma, \epsilon>0$, and $p \geq q$, such that for $g \in L^{2}(\Omega)$

$$
\left\|\exp \left\{-\epsilon \Delta t(-\Delta)^{p}\right\} g\right\|_{2} \leq \Gamma\left\|\exp \left\{-\omega \Delta t\left(L^{*} L\right)^{q / 2}\right\} g\right\|_{2},
$$

as postulated in Eq. (2.4). Then, $\|B\|_{2} \leq 1+\Delta t\left|\lambda_{Q}\right|$, the compensated explicit scheme Eq. (4.3) is unconditionally stable, and $v^{n}$ satisfies

$$
\left\|v^{n}\right\|_{2} \leq \exp \left\{\left|\lambda_{Q}\right| n \Delta t\right\}\|f\|_{2}, \quad n=0,1,2, \cdots, N_{T} .
$$

Proof : From Eq. (4.4) and Lemma 2, with $A$ as in Eq. (3.1),

$$
\begin{aligned}
\left\|v^{n+1}\right\|_{2} & =\left\|B v^{n}\right\|_{2} \leq\left\|S\left(v^{n}-\Delta t L v^{n}\right)\right\|_{2}=\left\|A v^{n}\right\|_{2} \\
& \leq \exp \left\{\left|\lambda_{Q}\right|(n+1) \Delta t\right\}\|f\|_{2}, \quad n=0,1,2, \cdots, N_{T} .
\end{aligned}
$$

QED. Using the analog of Lemma 3 for the compensating operator $R$, together with the argument in Theorem 1, leads to the corresponding result for the explicit scheme in Eq. (4.3).

THEOREM 2. Let $u^{n}$ be the unique solution of $u_{t}=-L u, u(0)=f$, at $t=n \Delta t$. Let $v^{n}$ be the corresponding solution of the $R$-compensated scheme in Eq. (4.3), and let $E R(t) \equiv u^{n}-v^{n}$, denote the error at $t=n \Delta t, \quad n=0,1,2, \cdots, N_{T}$. Then, with $p, q, Q, \Gamma, \epsilon$ as in Lemma 4 , and $\omega=\left|\lambda_{Q}\right|^{1-q}$,

$$
\begin{aligned}
\|E R(t)\|_{2} \leq & e^{\left|\lambda_{Q}\right| t}\|E R(0)\|_{2}+\left\{\left(e^{\left|\lambda_{Q}\right| t}-1\right) /\left|\lambda_{Q}\right|\right\} \times \\
& \left\{\epsilon \left|\left\|( - \Delta ) ^ { p } u \left|\left\|_{2, \infty}+\epsilon \Delta t\left|\left\|(-\Delta)^{p} L u\left|\left\|_{2, \infty}+1 / 2(\Delta t)\right\|\right| L^{2} u \mid\right\|_{2, \infty}\right\}\right.\right.\right.\right.\right. \\
(4.7) \quad= & e^{\left|\lambda_{Q}\right| t}\|E R(0)\|_{2}+\left\{\left(e^{\left|\lambda_{Q}\right| t}-1\right) /\left|\lambda_{Q}\right|^{q}\right\}(\epsilon / \omega)\left\|(-\Delta)^{p} u \mid\right\|_{2, \infty}+O(\Delta t) .
\end{aligned}
$$

Analogously to Eqs. (3.14), (3.18), we have the following Corollaries to Theorem 2.

COROllary 1. In Theorem 2, let $u_{t}=-L u, u(0)=f$, be a well-posed forward problem with data $f$ known exactly. Then, Eq. (4.7) reduces to

$$
\|E R(t)\|_{2} \leq\left\{\left(e^{\left|\lambda_{Q}\right| t}-1\right) /\left|\lambda_{Q}\right|^{q}\right\}(\epsilon / \omega)\left\|\mid(-\Delta)^{p} u\right\| \|_{2, \infty}+O(\Delta t) .
$$

Corollary 2. In Theorem 2, let $u_{t}=-L u, u(0)=f, 0 \leq t \leq T$, be an ill-posed backward problem with approximate input data $f$ and solution $u(t)$ satisfying

$$
\|E S(0)\|_{2} \leq \delta, \quad\|u(., T)\|_{2} \leq M,
$$


with known $M, \delta$. Then, choosing $\left|\lambda_{Q}\right|=(1 / T) \log (M / \delta)$, Eq. (4.7) reduces to

$$
\begin{aligned}
\|E R(t)\|_{2} & \leq M^{t / T} \delta^{(T-t) / T} \\
& +\left\{\left(e^{\left|\lambda_{Q}\right| t}-1\right) /\left|\lambda_{Q}\right|^{q}\right\}(\epsilon / \omega)\left\|(-\Delta)^{p} u \mid\right\|_{2, \infty}+O(\Delta t), \quad 0 \leq t \leq T .
\end{aligned}
$$

Remark 2. Laplacian stabilization and the operator $R$ requires the selection of $\epsilon>0, p>1$ in Eq. (4.2), and $\Gamma>0$ in Eq. (4.3), together with subsequent interactive adjustment of these parameters based on prior knowledge about the solution. Having located a useful triple $(\epsilon, p, \Gamma)$, an estimate of the stabilization penalty as $\Delta t \downarrow 0$ is not immediate in Eqs. (4.8), (4.10). Knowledge of the key parameters $\omega$ and $q$ in the governing inequality in Eq. (2.4) is needed to define $\left|\lambda_{Q}\right|=\omega^{1 /(1-q)}$, and evaluate the necessary terms in Eqs. (4.8), (4.10).

5. Fourier space Laplacian stabilization on rectangular regions. For simplicity of exposition we restrict attention to $R^{2}$ and functions $h(x, y)$, but the discussion is valid in higher dimensions. Define the $2 \mathrm{D}$ Fourier transform of an $L^{1}\left(R^{2}\right)$ function $h(x, y)$ by

$$
\hat{h}(\xi, \eta) \equiv \int_{R^{2}} h(x, y) e^{-2 \pi i(\xi x+\eta y)} d x d y .
$$

For fixed $p>1$, and small $\epsilon>0$, consider the well-posed forward diffusion initial value problem on the whole space $R^{2}$, involving possibly non-integer powers of the negative Laplacian,

$$
w_{t}=-\epsilon(-\Delta)^{p} w, \quad t>0, \quad w(x, y, 0)=h(x, y) .
$$

This has the unique Fourier space solution

$$
\hat{w}(\xi, \epsilon, t)=\exp \left\{-\epsilon t\left[(2 \pi \xi)^{2}+(2 \pi \eta)^{2}\right]^{p}\right\} \hat{h}(\xi, \eta), \quad t>0,
$$

from which $w(x, y, t)$ can be found by inverse Fourier transformation

$$
\text { (5.4) } w(x, y, t)=\int_{R^{2}} \exp \{2 \pi i(\xi x+\eta y)\} \exp \left\{-\epsilon t\left[(2 \pi \xi)^{2}+(2 \pi \eta)^{2}\right]^{p}\right\} \hat{h}(\xi, \eta) d \xi d \eta .
$$

Next, consider the parabolic initial value problem $u_{t}=-L u, t>0, u(0)=f$, on all of $R^{2}$, with the explicit scheme $u^{n+1}=u^{n}-\Delta t L u^{n}, n \geq 0$. Analogously to Eq. (4.2), we may define the compensating operator $R$ acting on $g(x, y) \in L^{1}\left(R^{2}\right) \cap L^{2}\left(R^{2}\right)$ by

$$
R g=\int_{R^{2}} \exp \{2 \pi i(\xi x+\eta y)\} \exp \left\{-\epsilon \Delta t\left[(2 \pi \xi)^{2}+(2 \pi \eta)^{2}\right]^{p}\right\} \hat{g}(\xi, \eta) d \xi d \eta
$$

Assume the inequality in Eq. (2.4) holds for $g(x, y) \in L^{1}\left(R^{2}\right) \cap L^{2}\left(R^{2}\right)$. With $\Gamma>0$ as in Eq. (2.4), this leads to the compensated explicit scheme on $0 \leq t \leq T$,

$$
\begin{aligned}
& v^{0}=f \\
& v^{n+1}=R\left\{v^{n}-\Delta t L v^{n}\right\} / \Gamma, \quad n=0,1,2, \cdots, N_{T}-1, \quad \Delta t=T / N_{T} .
\end{aligned}
$$


5.1. FFT periodized Laplacian stabilization and $I M \times I M$ images. Consider now the parabolic problem $u_{t}=-L u, t>0, u(0)=f$, defined on the unit square $\Omega$ in $R^{2}$, with $u(x, y, t)$ an 8 -bit $I M \times I M$ pixel image. We may discretize the elliptic spatial operator $L$ using centered finite differencing, with homogeneous Dirichlet or Neumann conditions on $\partial \Omega$. With pre-selected $\Gamma, \epsilon, p$, we then apply the compensated explicit scheme in Eq. (5.6) above as follows. At each time step, direct and inverse Fast Fourier Transform (FFT) algorithms are used to synthesize $R g$ in Eq. (5.5), where $g$ is the $I M \times I M$ pixel image $g=\left(v^{n}-\Delta t L v^{n}\right\} / \Gamma$. However, the FFT algorithm assumes $g$ to be extended by periodicity to all of $R^{2}$, and returns an $I M \times I M$ array $v^{n+1}=R g$ satisfying periodic boundary conditions. At the next time step, application of the discretized operator $L$ to $v^{n+1}$ restores the original Dirichlet or Neumann boundary conditions. In practice, in many problems of interest, such alternating erroneous boundary conditions are found to cause spurious artifacts at the very edges of the image, without impairing the results away from the edges. Such incovenience is a small price to pay for the highly efficient FFT synthesis of the compensator $R$, which enables consideration of $512 \times 512$ or $1024 \times 1024$ arrays. This technique can clearly be extended to nonlinear parabolic equations on hyperrectangles in $R^{n}$.

6. Van Cittert iteration and backward continuation in $u_{t}=-L u$. As originally formulated [24], the Van Cittert iterative procedure was intended for solving 1D linear convolution integral equations $P w=f$ with explicitly known kernels, by means of the iterative procedure

$$
w^{m+1}(x)=w^{m}(x)+\lambda\left\{f(x)-P\left[w^{m}(x)\right]\right\}, \quad m \geq 1 .
$$

Here, $\lambda>0$ is a fixed relaxation parameter, $w^{1}(x)=\lambda f(x)$, and the expectation is that $w^{m} \rightarrow w$. In reality, ill-posedness of the integral equation leads to error amplification at high frequencies which prevents convergence. However, in spectroscopy and image processing applications [9], [12], the Van Cittert method is often found to produce useful results after finitely many iterations, even though it may not converge.

Application of the Van Cittert iteration to nonlinear parabolic backward continuation is discussed in [7]. Consider the well-posed forward nonlinear problem $u_{t}=-L u, u(0)=f, 0 \leq t \leq T$, in a domain $\Omega \in R^{n}$. Let $\Lambda^{T}$ be the nonlinear solution operator at time $T$ so that $\Lambda^{T}[u(0)]=u(T)$. The Van Cittert procedure would seek to recover the initial value $f=u(0)$, from approximate data $g$ for $u(T)$, through the iteration

$$
w^{m+1}=w^{m}+\lambda g-\Lambda^{T}\left[w^{m}\right], \quad m \geq 1,
$$

with $w^{1}=\lambda g$, and fixed relaxation parameter $\lambda, 0<\lambda<1$. By discretizing the forward problem $u_{t}=-L u, u(0)=f, 0 \leq t \leq T$, using a stable computational method, we may define a corresponding discrete nonlinear solution operator $\Lambda_{d}^{T}$, and use it in Eq. (6.2) to implement the procedure computationally. Several examples of successful nonlinear backward recovery using this technique are presented in [7].

7. 2D nonlinear computational examples. The illustrative examples below involve two nonlinear second order parabolic equations on the unit square $\Omega \equiv\{0 \leq$ $x, y \leq 1\}$ in $R^{2}$, with homogeneous Neumann boundary conditions. The initial values $w(x, y, 0)=g(x, y)$, are 8 bit grey scale images with intensity values ranging from 0 to 255 , leading to sizeable nonlinearities involving $w$. All images are of size $512 \times 512$ 
pixels, and are associated with complicated intensity surface plots such as are shown in Figure 1.1. One equation has the form

$$
w_{t}=\kappa s(w) \nabla \cdot\{q(x, y, t) \nabla w\}+c \sqrt{|w|} w_{x}+d\left(w \cos ^{2} w\right) w_{y}, \quad \Omega \times(0, T),
$$

$$
w(x, y, 0)=g(x, y)
$$

where $\kappa=8.5 \times 10^{-4}, c>0, d>0$, and

$$
\begin{aligned}
& s(w)=\exp (0.002 w), \\
& q(x, y, t)=\exp (10 t)(1+5 \sin \pi x \sin \pi y) \geq 1, \quad \Omega \times(0, T) .
\end{aligned}
$$

The other equation used differs from the above by having the term $c w w_{x}$ in place of the term $c \sqrt{|w|} w_{x}$. Clearly, the nonlinear elliptic operator $\widetilde{L}$ in Eq. (7.1) is very far from the linear $L$ contemplated in the assumed inequality Eq. (2.4). Numerous other interesting nonlinear parabolic equations remain to be explored.

Discretization of Eq. (7.1) uses centered finite differencing for the space variables with $\Delta x=\Delta y=1 / 512$, together with pure explicit time differencing, with $\Delta t>0$ chosen so as to yield useful results.

8. Well-posed forward experiment. The first experiment illustrates the usefulness of the stabilized explicit scheme in forward solving Eq. (7.1) up to time $T=0.004$, with $c=0.0625, d=0.075$. With $\Delta t=0.0002$, the uncompensated pure explicit scheme applied to the $512 \times 512$ pixel Sydney and B2 Bomber images develops instabilities, as shown in the middle images in Figure 8.1. However, applying FFT Laplacian stabilization as discussed in Section 5, using $\Gamma=1.0, \epsilon=4.0 \times 10^{-6}, p=$ 2.5 , produces useful results with the same value of $\Delta t$. This is shown in the rightmost images in Figure 8.1. This particular $(\epsilon, p, \Gamma)$ triple was arrived at after very few trials. Note the FFT-induced edge effects at top and bottom of the stabilized images.

For comparison, an uncompensated stable pure explicit calculation was also performed, with $\Delta t$ chosen ten times smaller. Slightly different results were obtained at $T=0.004$. These differences amounted to an $L^{1}$ relative error of $9.26 \%$ for the Sydney image, and $3.87 \%$ for the B2 Bomber image. Such $L^{1}$ errors indicate relatively small stabilization penalties in these two examples.

It is noteworthy that the linear analysis provided in Sections 2 through 5 , together with the assumed inequality in Eq. (2.4), appear to be applicable in this nonlinear case. The FFT compensated explicit scheme becomes ever more useful in well-posed forward 3D problems on finer spatial meshes.

9. Ill-posed backward recovery experiments. While the forward computational results are encouraging, successful backward reconstruction in nonlinear problems is considerably more difficult, as the accuracy of the input data at time $T$ plays a crucial role. For linear autonomous selfadjoint forward parabolic equations $u_{t}=-L u, 0 \leq t \leq T$, approximate data $g$ at time $T$ such that $u(T)-g{ }_{2} \leq \delta$, for a solution $u(t)$ known to satisfy $u(0){ }_{2} \leq M$, leads to an $L^{2}$ uncertainty of $M^{(T-t) / T} \delta^{t / T}$ in backward reconstruction at time $t<T$. See [10], [15].

However, if $L$ is nonlinear, the corresponding $L^{2}$ uncertainty at time $t<T$ may be larger [10]. It is given by $M^{1-\mu(t)} \delta^{\mu(t)}$, where the Hölder exponent $\mu(t)$ satisfies $0 \leq \mu(t) \leq 1$, with $\mu(t)>0, t>0, \mu(T)=1, \mu(0)=0$, and $\mu(t) \downarrow 0$ monotonically as $t \downarrow 0$. Rapid exponential decay of $\mu(t$ to zero as $t \downarrow 0$, is possible in some cases. 
STABLE EXPLICIT TIME-MARCHING IN NONLINEAR PARABOLIC EQUATIONS 13
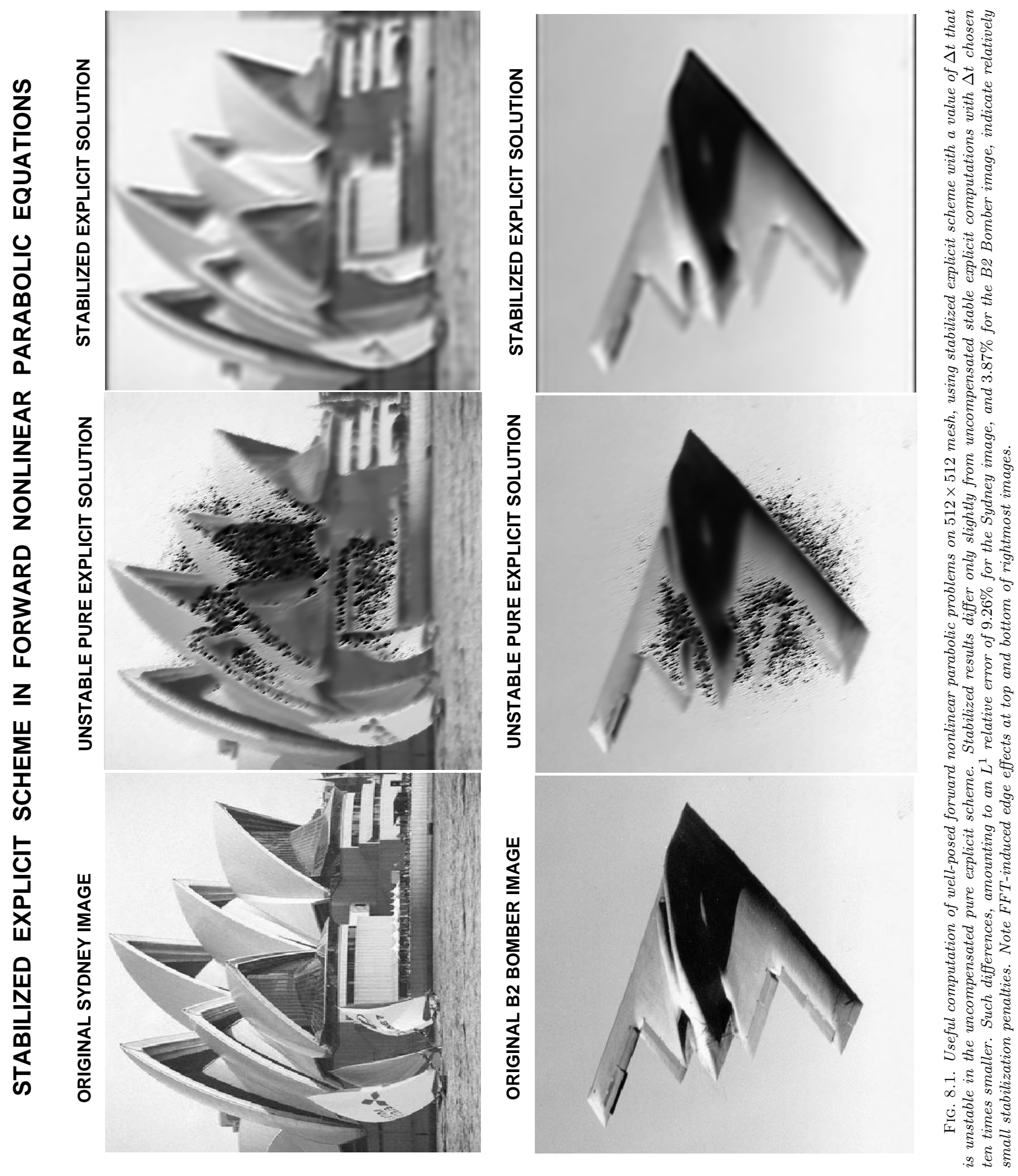
For this reason, high quality nonlinear reconstructions from imprecise data are not generally feasible. See [6].

In the examples below, the input data at time $T$ is obtained by a preliminary forward solution of the nonlinear parabolic equation, using a stable numerical method. That numerically obtained blurred image may not be a sufficiently accurate approximation to the true solution of Eqs. (7.1) or (9.1), at time $T$. In some cases, recovering the finest details in the sharp image at $t=0$ from such modestly accurate data at $t=T$, may lie beyond the capabilities of any method on theoretical grounds alone.

Sagittal MRI brain scan. The original sharp MRI brain image in Figure 9.1 was blurred by using it as initial data in Eq. (7.1) with $c=0.05, d=0.06$, and applying the stable uncompensated pure explicit scheme with $\Delta t=1.75 \times 10^{-6}$, to march forward 2000 time steps up to the final time $T=3.5 \times 10^{-3}$. This produced the blurred image in Figure 9.1. While this is a reasonably accurate computation, a great deal of fine detail in the sharp image has been smoothed out by the nonlinear evolution, as becomes evident at high magnification.

The numerically blurred image was used as input data in the FFT Laplacianstabilized explicit scheme discussed in Section 5, using $\Gamma=1.0, \epsilon=2.0 \times 10^{-8}, p=$ 2.75. With $\Delta t=1.75 \times 10^{-6}$, the compensated scheme was marched 2000 time steps backward from $T=3.5 \times 10^{-3}$, to produce the deblurred image shown at the bottom left of Figure 9.1. Clearly, significant deblurring was achieved, and the linear analysis in Sections 2 through 5, together with Eq. (2.4), again appear to be germane in this nonlinear situation. Here, the small stabilization penalty is added to the nonlinear $L^{2}$ uncertainty, $M^{1-\mu(t)} \delta^{\mu(t)}$, with an unknown $\mu(t)$.

The Van Cittert iterative procedure discussed in Section 6 was succesfully applied to several nonlinear examples in [7], although at substantially higher computational costs. Significantly, this procedure fails in the present example, as shown at the bottom right image in Figure 9.1. Inverse reconstruction methods generally work well only in limited cases, and alternative approaches, when available, are valuable.

It is instructive to view the above reconstruction process as it operates on the actual image intensity data that is fed into the computational algorithm. This is shown in Figure 9.2. Clearly, quite complex data surfaces are involved, and accurate recovery would seem quite difficult. Inspection of the bottom two reconstructed surfaces in Figure 9.2 reveals how easily the process can go awry.

Elizabeth Taylor image. A familiar face image makes a valuable test example. The original $512 \times 512$ pixel Elizabeth Taylor image in Figure 9.3 was blurred by using it as initial data in the following equation on the unit square $\Omega$

$$
\begin{aligned}
& w_{t}=\kappa s(w) \nabla \cdot\{q(x, y, t) \nabla w\}+c w w_{x}+d\left(w \cos ^{2} w\right) w_{y}, \quad \Omega \times(0, T), \\
& w(x, y, 0)=g(x, y),
\end{aligned}
$$

with $\kappa$ and $q(x, y, t)$ as in Eq. (7.1), $c=0.05$ and $d=0.06$. The stable uncompensated pure explicit scheme was applied with $\Delta t=7.0 \times 10^{-6}$ and 600 time steps up to the final time $T=4.2 \times 10^{-3}$. With this larger $\Delta t$, the resulting numerical solution at time $T$ is a less accurate approximation to the true solution than was the case in Figure 9.1.

The stronger nonlinearity associated with the $w w_{x}$ term leads to the striking transformation shown in the resulting blurred image in Figure 9.3. Such distortion 


\section{STABILIZED EXPLICIT SCHEME IN BACKWARD NONLINEAR PARABOLIC EQUATIONS}

ORIGINAL MRI BRAIN SCAN

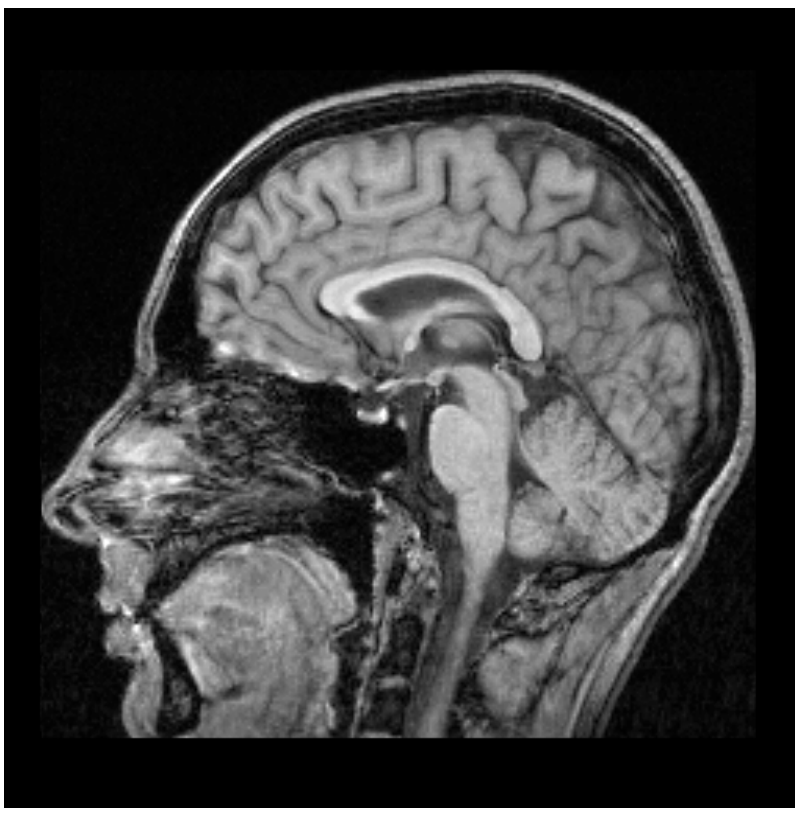

STABILIZED EXPLICIT DEBLUR

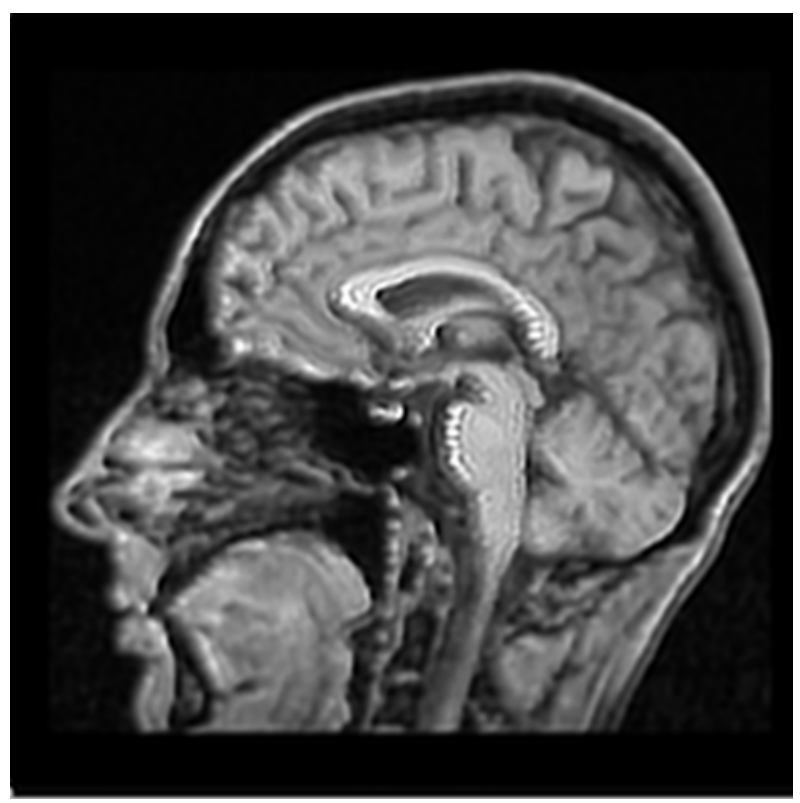

NONLINEARLY BLURRED IMAGE

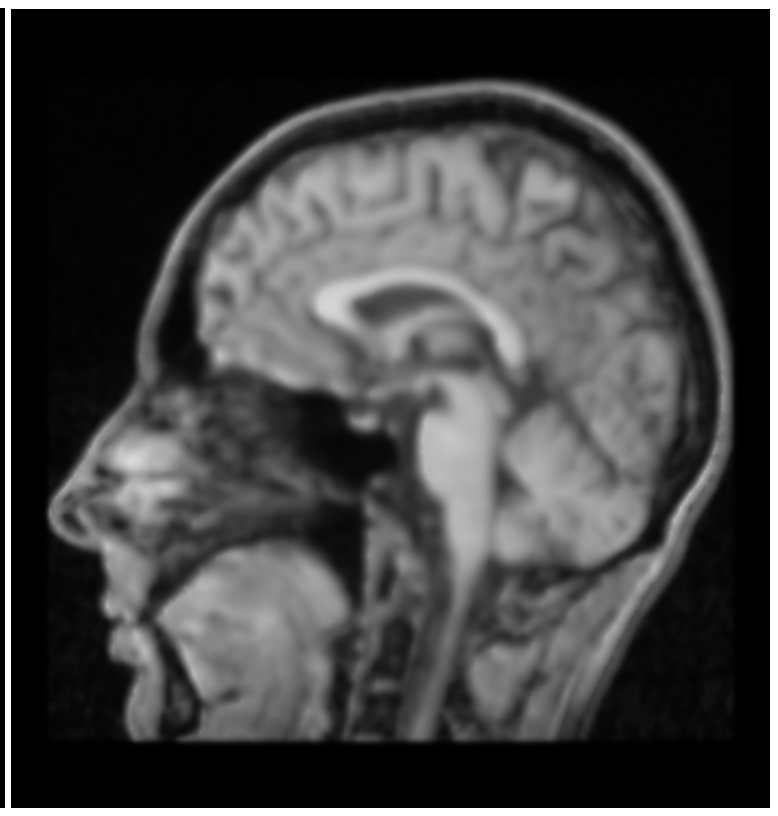

VAN CITTERT ITERATIVE DEBLUR

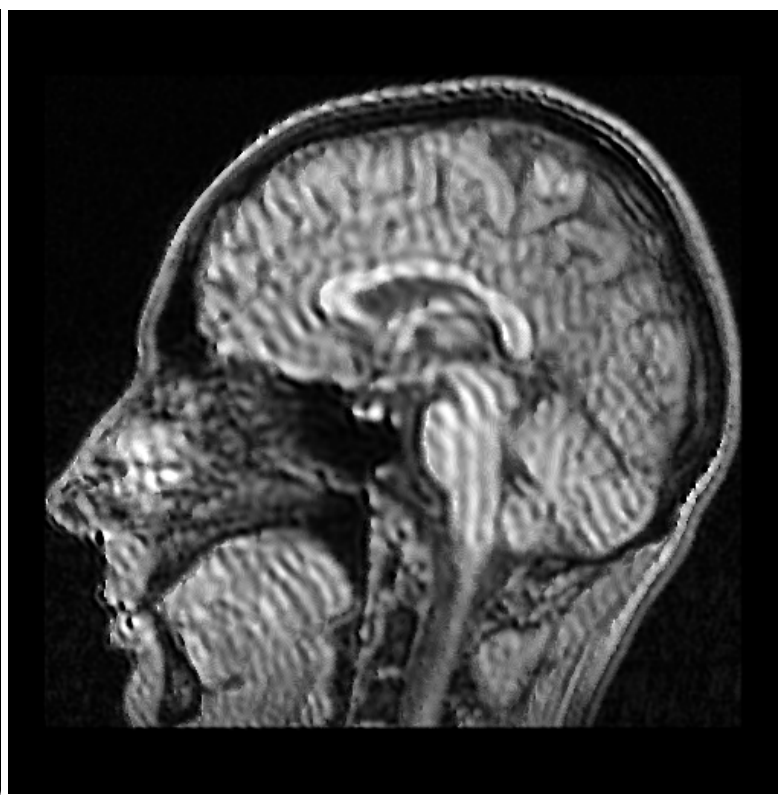

FIG. 9.1. Backward recovery from nonlinearly blurred data in $512 \times 512$ MRI brain image, using stabilized explicit scheme with $\Gamma=1.0, \epsilon=2.0 \times 10^{-8}, p=2.75$. Van Cittert procedure develops instabilities after 30 iterations. 


\section{STABILIZED EXPLICIT RECONSTRUCTION OF MRI BRAIN SCAN INTENSITY SURFACE}

ORIGINAL MRI BRAIN SCAN

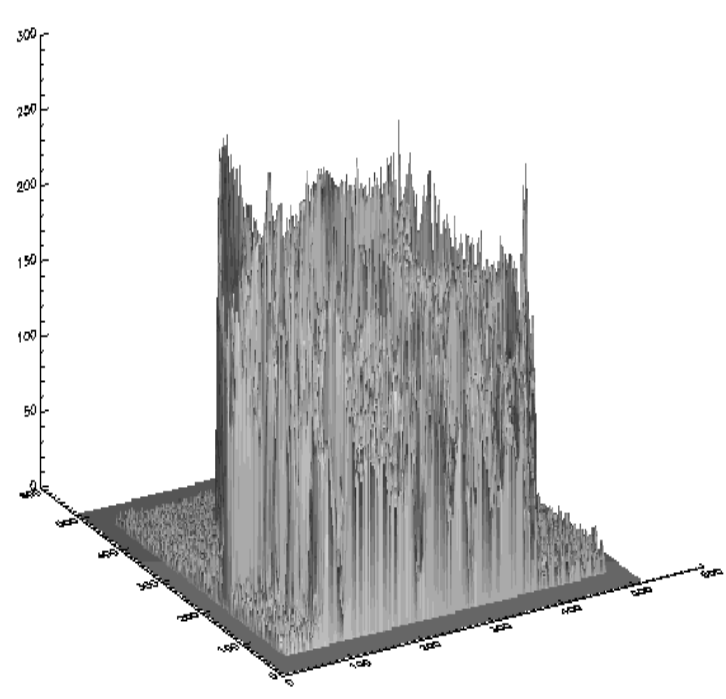

STABILIZED EXPLICIT DEBLUR

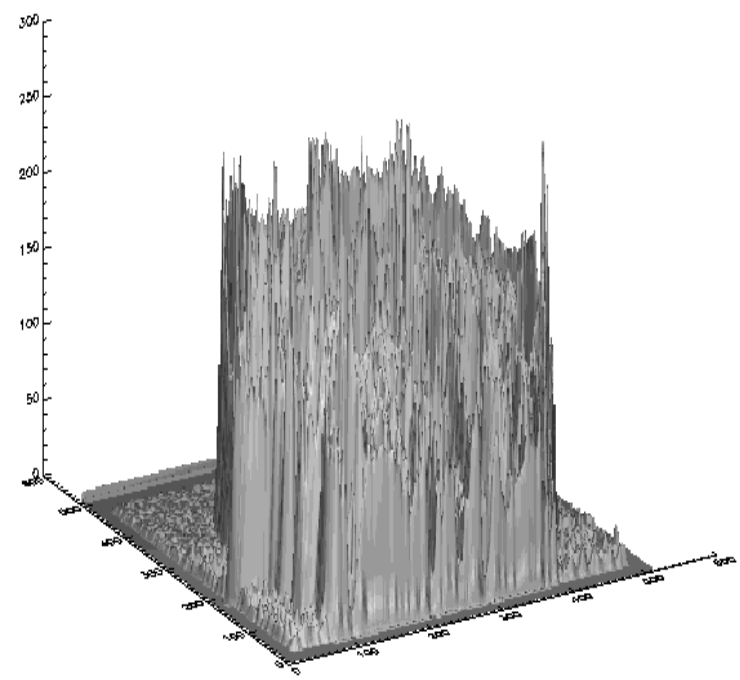

NONLINEARLY BLURRED IMAGE

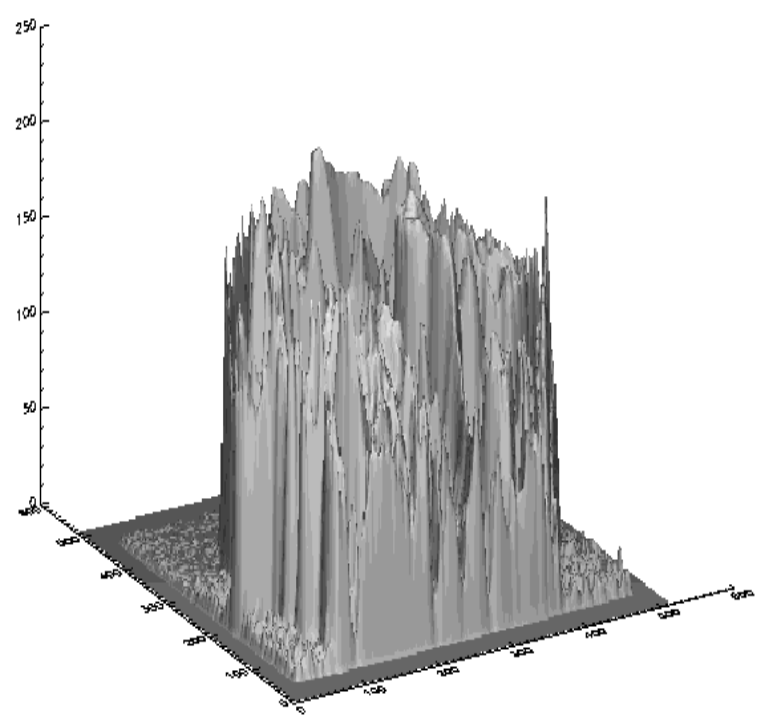

VAN CITTERT ITERATIVE DEBLUR

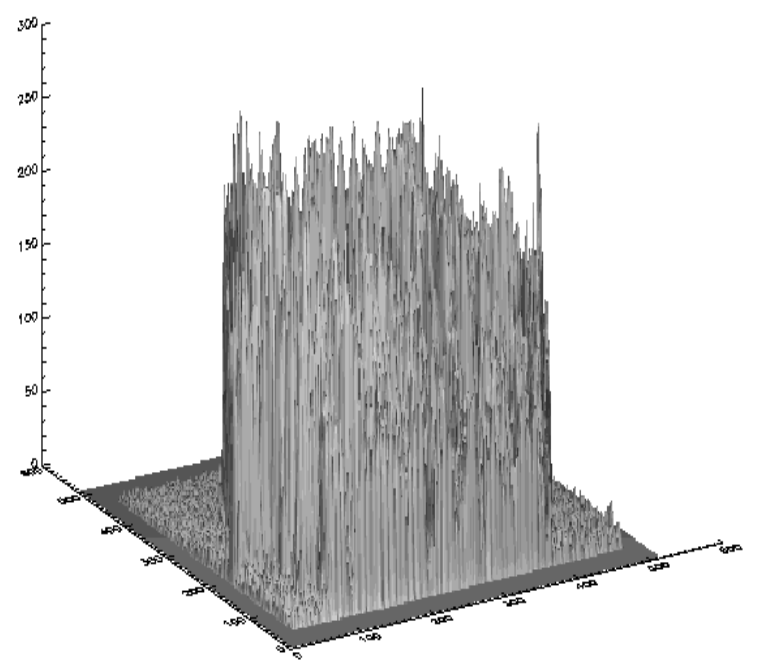

FIG. 9.2. Reconstruction of highly irregular original intensity surface from blurred data in MRI brain image, using stabilized explicit scheme and Van Cittert procedure. 


\section{STABILIZED EXPLICIT SCHEME IN BACKWARD NONLINEAR PARABOLIC EQUATIONS}

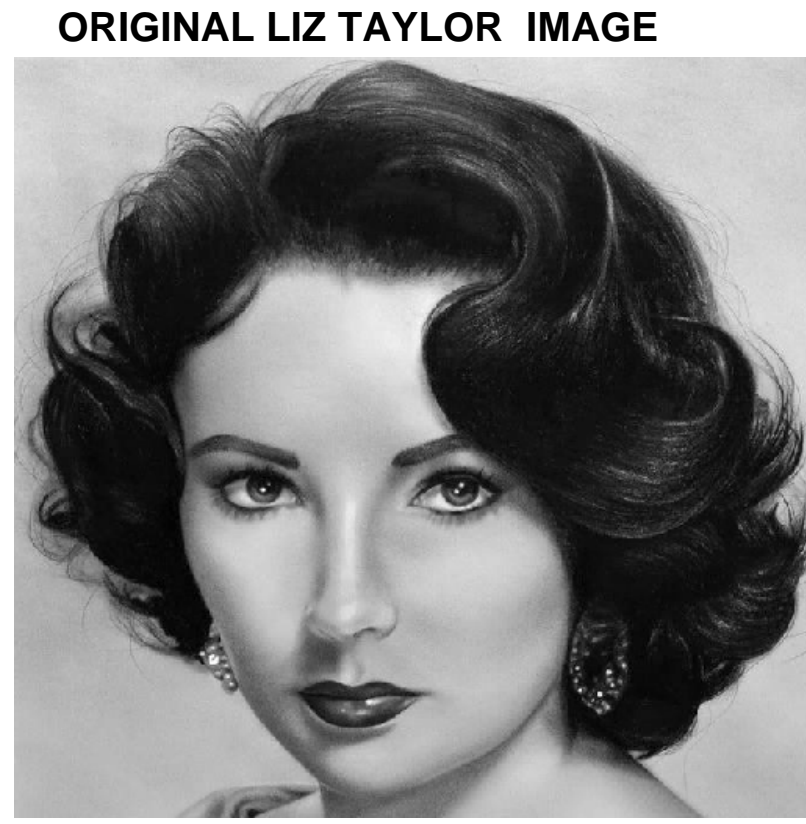

NONLINEARLY BLURRED IMAGE

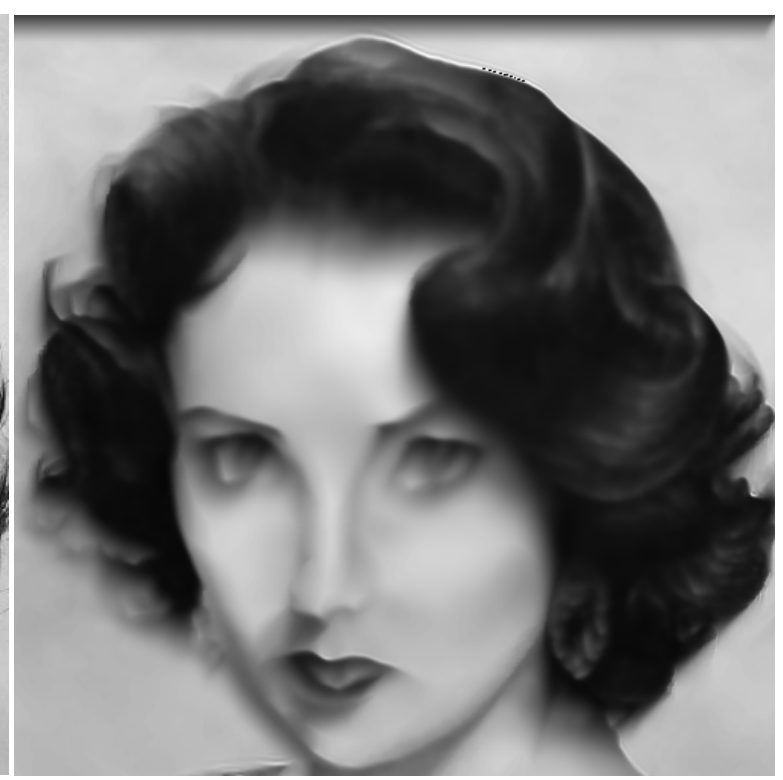

\section{STABILIZED EXPLICIT DEBLUR}

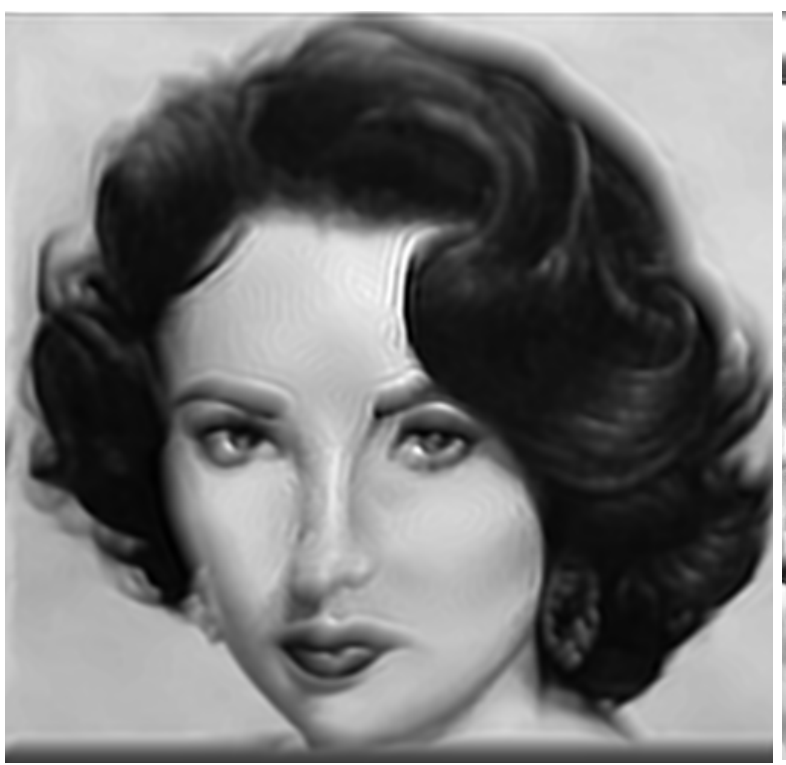

VAN CITTERT ITERATIVE DEBLUR

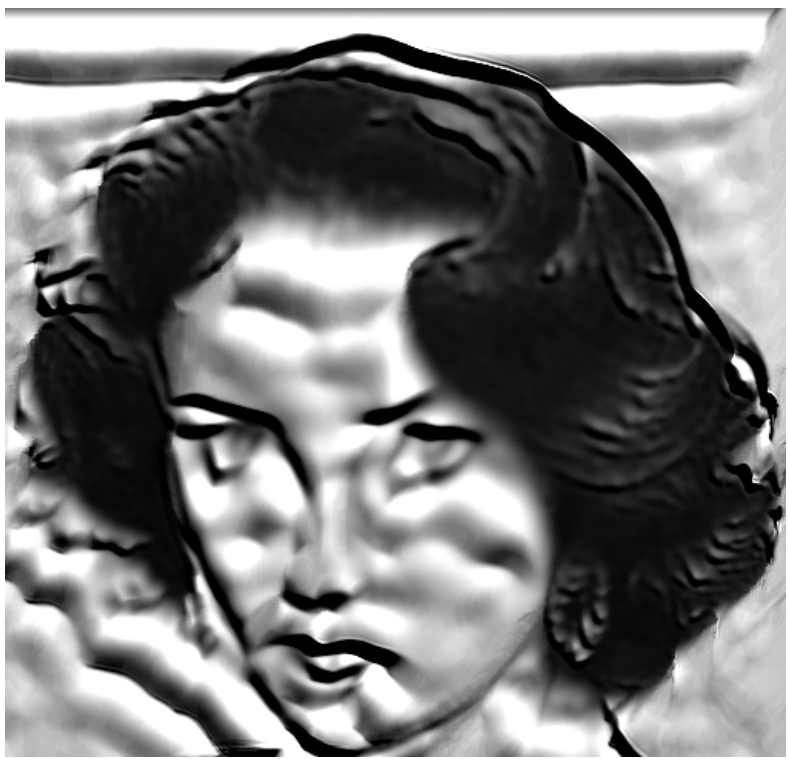

FIG. 9.3. Backward recovery from nonlinearly blurred data in $512 \times 512$ Liz Taylor image, using stabilized explicit scheme with $\Gamma=1.0, \epsilon=7.5 \times 10^{-8}, p=2.735$. Van Cittert procedure develops instabilities after 8 iterations 


\section{STABILIZED EXPLICIT RECONSTRUCTION OF LIZ TAYLOR IMAGE INTENSITY SURFACE}

ORIGINAL LIZ TAYLOR IMAGE

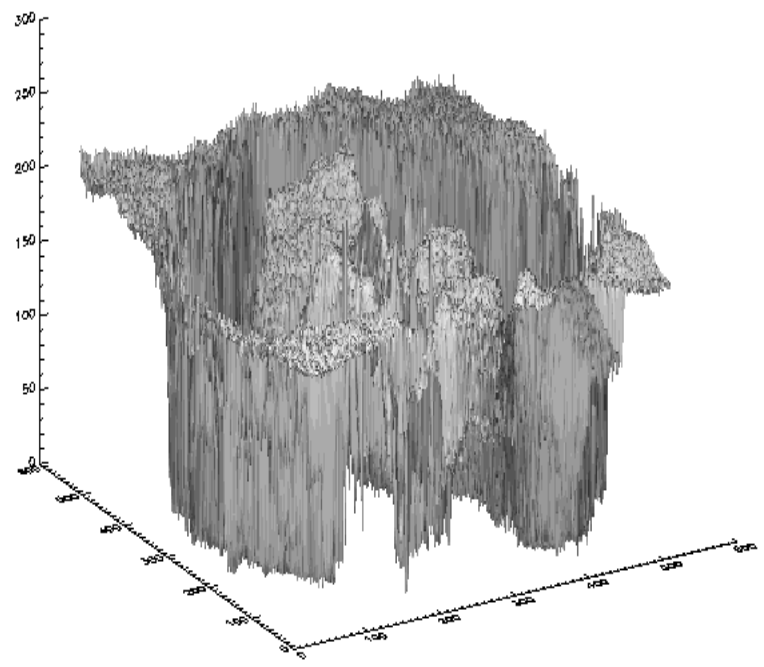

STABILIZED EXPLICIT DEBLUR

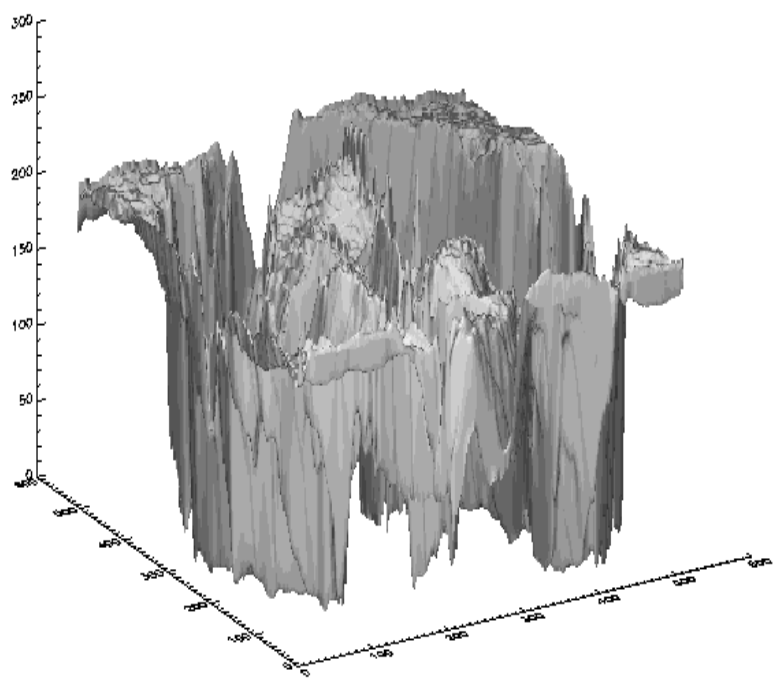

NONLINEARLY BLURRED IMAGE

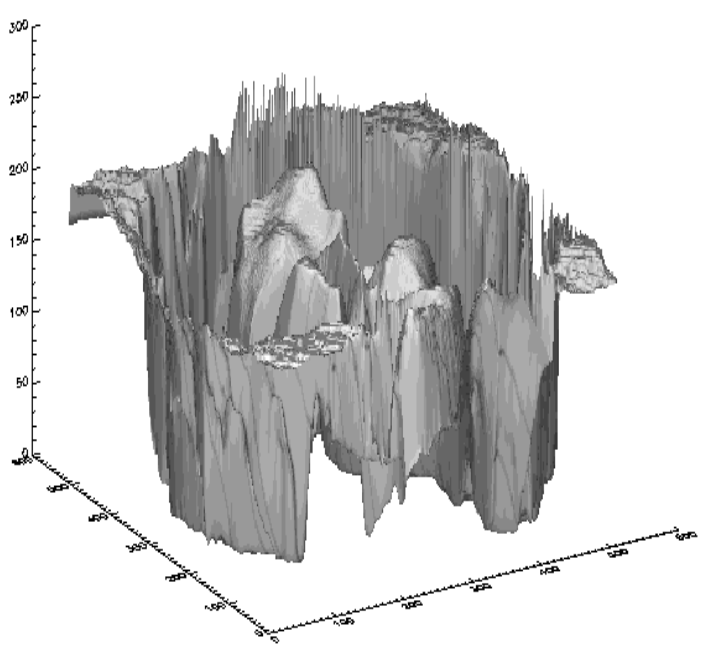

VAN CITTERT ITERATIVE DEBLUR

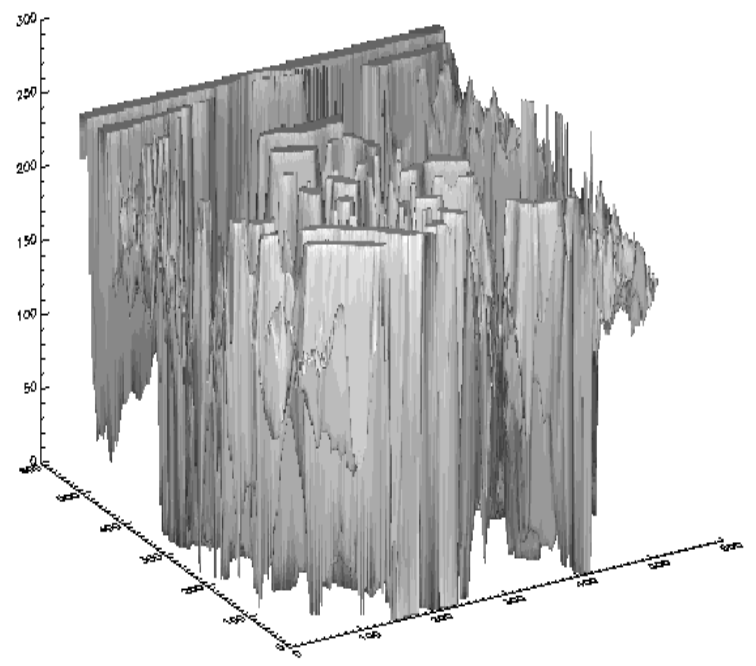

FIG. 9.4. Reconstruction of highly irregular original intensity surface from blurred data in Liz Taylor image, using stabilized explicit scheme and Van Cittert procedure. 
is not easily achieved using traditional convolution with a Gaussian point spread function. Recovery of the original from such a strongly blurred image, computed with only modest accuracy, seems less feasible than was possible in the MRI image. Surprisingly, using $\Gamma=1.0, \epsilon=7.5 \times 10^{-8}, p=2.735$, and marching back 600 time steps $\Delta t=7.0 \times 10^{-6}$ from $t=T$ in the stabilized explicit scheme, produced the reasonably good reconstruction shown in Figure 9.3. This is a significant example. Clearly, the stabilization penalty must be quite small to permit such useful recovery in the presence of the nonlinear $L^{2}$ uncertainty $M^{1-\mu(t)} \delta^{\mu(t)}$.

The poor performance of the Van Cittert iteration in Figure 9.3 is a reflection of the particularly difficult backward continuation problem associated with the blurred Elizabeth Taylor image, due to the nonlinearity in Eq. (9.1). The reconstruction process on the corresponding intensity surface data is displayed in Figure 9.4.

10. Relation to the quasi-reversibility method. With its emphasis on solving multidimensional nonlinear problems using explicit schemes, the present method is a significant new approach that complements the quasi-reversibility (QR) method [13]. Applications of the QR method to backward parabolic equations are developed in [13, Chapter 1]. There, linear equations in the form $w_{t}+A(t) w=0$, are considered, with $A(t)$ a linear elliptic partial differential operator of order $2 m$ with coefficients depending smoothly on space and time, and such that the forward problem is well-posed. Backward continuation from given noisy data at time $T>0$, is accomplished by numerically marching back from $t=T$ in the modified equation $w_{t}+A(t) w-\omega A^{*}(t) A(t) w=0$, with suitably pre-selected small $\omega>0$. This modified equation is well-posed backward in time, and a variety of stable difference schemes may be used. As formulated in [13, Chapter 1], the QR method is not applicable to nonlinear problems in an obvious way, due to the difficulty of defining a suitable adjoint operator $A^{*}(t)$. Indeed, linear problems are the primary focus in [13]. In addition, implicit Crank-Nicolson time differencing is contemplated for the numerical solution of the modified higher order equation involving $A^{*}(t) A(t)$. In multidimensional problems, this requires computationally intensive solution of the algebraic problem at each time step. This is avoided in the present method.

A valuable feature of the stabilized explicit scheme is that it allows for efficient and simultaneous exploration of the parameter values $(\Gamma, \epsilon, p)$, including non integer positive values of $p$ in $(-\Delta)^{p}$, all within the same computational code. In the $\mathrm{QR}$ method for second order parabolic equations, the modified equation involves a fixed fourth order stabilizing elliptic operator, and there is no option for exploring other stabilizers. In this connection, it is noteworthy that in both the well-posed and illposed test examples in Sections 8 and 9, values of $p$ such that $2.5 \leq p \leq 2.75$, were arrived at interactively. Apparently, a compensating pseudo-diffusion equation of order five or higher, seems to be necessary to control explicit scheme instability in computing second order nonlinear parabolic equations.

11. Concluding Remarks. The unexpected success of the FFT Laplacianstabilized explicit scheme on 2D nonlinear parabolic problems in rectangular regions, indicates that the use of Laplace stabilization in other special domains in $R^{n}$ where Laplacian eigenfunctions are known in closed form, is worthy of active consideration. More generally, in arbitrary domains $\Omega \in R^{n}$ as envisaged in Eq. (3.20), preliminary computation of a sufficient number of the characteristic pairs of a variable coefficient linear elliptic operator $L$, believed to be representative of the given nonlinear elliptic operator $\widetilde{L}$, may provide the key to useful stabilized explicit solution of difficult nonlinear parabolic problems. 
The apparent validity of Eq. (2.4) in the numerical experiments in Sections 8 and 9 , raises interesting analytical research problems.

The failure of the Van Cittert method in the two examples in Section 9, despite its successful use in [7], indicates a continuing need for alternative reconstruction procedures, based on different analytical strategies.

The use of fictitious images in exploring 2D nonlinear parabolic equations, together with displays of the corresponding intensity surfaces, was particulary instructive.

Future work will explore the use of compensated explicit schemes in non-parabolic irreversible systems occurring in viscoelasticity, thermoelasticity, coupled sound and heat flow, and other areas of application discussed in [13].

\section{REFERENCES}

[1] W. Arendt And A. F. M. Ter Elst, Gaussian estimates for second order elliptic operators with boundary conditions, J. Operator Theory 38 (1997), pp. 87-130.

[2] D. G. Aronson, Bounds for the fundamental solution of a parabolic equation, Bull. Amer. Math. Soc. 73 (1967), pp. 890-896.

[3] J. Atmadja And A. C. Bagtzoglou, State of the art report on mathematical methods for groundwater pollution source identification, Environmental Forensics 2 (2001), pp. 205214.

[4] J. Atmadja And A. C. Bagtzoglou, Pollution source identification in heterogeneous porous media, Water Resources Research 37 (2001), p. 2113-2125. DOI 10.1029/2001WR000223

[5] I. BABUŠKA AND J. E. OsBorn, Finite element-Galerkin approximation of the eigenvalues and eigenvectors of selfadjoint problems, Math. Comp. 52 (1989), pp. 275-297.

[6] A. S. CARASSO, Reconstructing the past from imperfect knowledge of the present: Effective non-uniqueness in solving parabolic equations backward in time, Mathematical Methods in the Applied Sciences (2012), DOI: 10.1002/mma.2582

[7] A. S. CARAsso, Compensating operators and stable backward in time marching in nonlinear parabolic equations, Int J Geomath 5 (2014), pp. 1-16.

[8] V. Heuveline, On the computation of a very large number of eigenvalues for selfadjoint elliptic operators by means of multigrid methods, Journal of Computational Physics 184 (2003), pp. 321-337.

[9] P. A. Jansson, Deconvolution with Application in Spectroscopy, Academic Press, New York, 1984.

[10] R. J. KNops, Logarithmic convexity and other techniques applied to problems in continuum mechanics, in Symposium on Non-Well-Posed Problems and Logarithmic Convexity, R. J. Knops, ed., Lecture Notes in Math. 316, Springer-Verlag, New York, 1973.

[11] J. R. Kuttler And V. G. Sigillito, Eigenvalues of the Laplacian in two dimensions, SIAM Review 26 (1984), pp. 163-193.

[12] R. L. LAGENDiJK And J. Biemond, Iterative Identification and Restoration of Images, Kluwer Academic Publishers, Boston, 1991.

[13] R. Lattès And J. L. Lions, The Method of Quasi-Reversibility, American Elsevier, New York, 1969.

[14] J. D. Logan, Transport Modeling in Hydrogeochemical Systems, Springer, New York, 2001.

[15] K. Miller, Least squares methods for ill-posed problems with a prescribed bound, SIAM J. Math. Anal. 1 (1970), pp. 52-74.

[16] P. M. Morse And H. Feshbach, Methods of Theoretical Physics, Mc Graw-Hill, New York, (1953).

[17] R. M. Neupauer, B. Borchers, and J. L. Wilson, Comparison of inverse methods of reconstructing the release history of a groundwater contamination source, Water Resources Research 36 (2000), pp. 2469-2475. DOI 10.1029/95WR02383.

[18] E. M. Ouhabaz, Gaussian estimates and holomorphy of semigroups, Proc. Amer. Math. Soc. 123 (1995), pp. 1465-1474.

[19] E. M. Ouhabaz, Gaussian upper bounds for heat kernels of second order elliptic operators with complex coefficients on arbitrary domains, J. Operator Theory 51 (2004), pp. 335-360. 
[20] R. B. Platte And T. A. Driscoll, Computing eigenmodes of elliptic operators using radial basis functions, Computers and Mathematics with Applications 48 (2004), pp. 561-576.

[21] R.D. Richtmyer and K. W. Morton, Difference Methods for Initial Value Problems, Second Edition, Wiley, New York, 1967.

[22] R. N. Singh, Advection diffusion equation models in near-surface geophysical and environmental sciences, J. Ind. Geophys. Union 17 (2013), pp. 117-127.

[23] T. H. Skaggs And Z. J. Kabala, Recovering the history of a groundwater contaminant plume: Method of Quasi-Reversibility, Water Resources Research, 31 (1995), pp. 2669-2673. DOI 10.1029/95WR02383.

[24] P. H. VAN CitTERT, Zum Einfluss der Spaltbreite auf die Intensittsverteilung in Spektrallinien II, Z. Physik 69 (1931), pp. 298-308. 